UVX 2012, 01010 (2013)

DOI: $10.1051 /$ uvx/201301010

(C) Owned by the authors, published by EDP Sciences, 2013

\title{
Cinétique et mécanisme de croissance de nanoparticules d'or suivis par UV SAXS and XANES
}

\author{
O. Spalla ${ }^{1}$, F. Testard ${ }^{1}$, J. Han $^{1}$, B. Abécassis ${ }^{2}$ et Q. Kong ${ }^{3}$ \\ ${ }^{1}$ CEA Saclay, Laboratoire Interdisciplinaire sur l'Organisation Nanométrique et \\ Supramoléculaire, SIS2M UMR 3299, 91191 Gif-sur-Yvette Cedex, France \\ ${ }^{2}$ Laboratoire de Physique des Solides, UMR 8502, Université Paris Sud, 91405 Orsay \\ Cedex A, France \\ ${ }^{3}$ Synchrotron SOLEIL, Orme des Merisiers, BP. 48, 91192 Gif-sur-Yvette Cedex, France
}

\begin{abstract}
Résumé. Les applications des nanoparticules d'or demandent un contrôle précis de leurs caractéristiques morphologiques. L'obtention de ces nanoparticules peut s'effectuer en solution par la réduction d'une solution de sel d'or de type $\mathrm{HAuCl} 4$. Ces réactions peuvent être soit lentes (20 minutes) soit très rapides et parfois terminées en quelques dizaines de millisecondes. La nucléation et la croissance de ces objets nanométriques en solutions peuvent être suivies par différentes sondes sensibles à la distribution de taille et forme des objets ainsi qu'à la spéciation des atomes répartis entre solution et nanoparticules. Dans le cas des nanoparticules d'or, le couplage des spectroscopies UV et d'absorption X avec la diffusion aux petits angles des rayons $\mathrm{X}$ permet de comprendre les mécanismes intervenants lors de la réduction du sel d'or initial par différents réducteurs, soit forts (borohydrure dans le toluène), doux (acide ascorbique dans une solution aqueuse de CTABr), ou semi doux (acide ascorbique en excès dans l'eau). La forme et la taille des nanoparticules obtenues dépendent fortement des concentrations de réactifs et de $\mathrm{pH}$. Différents cas sont discutés dans cet article. L'intérêt et l'efficacité du couplage de ces mesures cinétiques expérimentales avec un modèle de croissance numérique est également discuté.
\end{abstract}

\section{INTRODUCTION}

Le développement actuel des nanotechnologies repose en partie sur la production de nanoparticules bien calibrées en forme et en taille, leur assemblage et fonctionnalisation [1]. On s'intéresse dans les travaux décrits ci-après à la première phase de ces différents challenges. Depuis le temps des « colloides », la synthèse de nanoparticules par voie solvant est essentiellement un domaine de la chimie des solutions où la réflexion est guidée par l'empirisme et la méthode d'essais erreurs. Depuis quelques années cependant, la volonté de suivre en détail les cinétiques de nucléation et croissance de nanoparticules s'est affirmée d'autant plus fortement que la brillance des rayonnement de synchrotron de $3^{\text {ème }}$ génération permet d'obtenir des résolutions temporelles comparables au cinétique de synthèses rapides [2-6].

\section{MÉTHODES EXPÉRIMENTALES}

Depuis quelques années, les mécanismes de nucléation et croissance des nanoparticules sont suivies en couplant différentes techniques offertes sur synchrotron [7,8]. Dans le cas des nanoparticules d'or, nous avons couplé principalement trois techniques de caractérisation, à savoir l'absorption UV-Visible, l'absorption des rayons et la diffusion centrale des rayons X. Dans tous les cas, ces méthodes de caractérisation in situ ont été effectuées en ligne au travers d'un capillaire en quartz où la solution réactive était injectée dans des conditions définies. Ces conditions étaient obtenues soit par un mélange

This is an Open Access article distributed under the terms of the Creative Commons Attribution License 2.0, which permits unrestricted use, distribution, and reproduction in any medium, provided the original work is properly cited. 


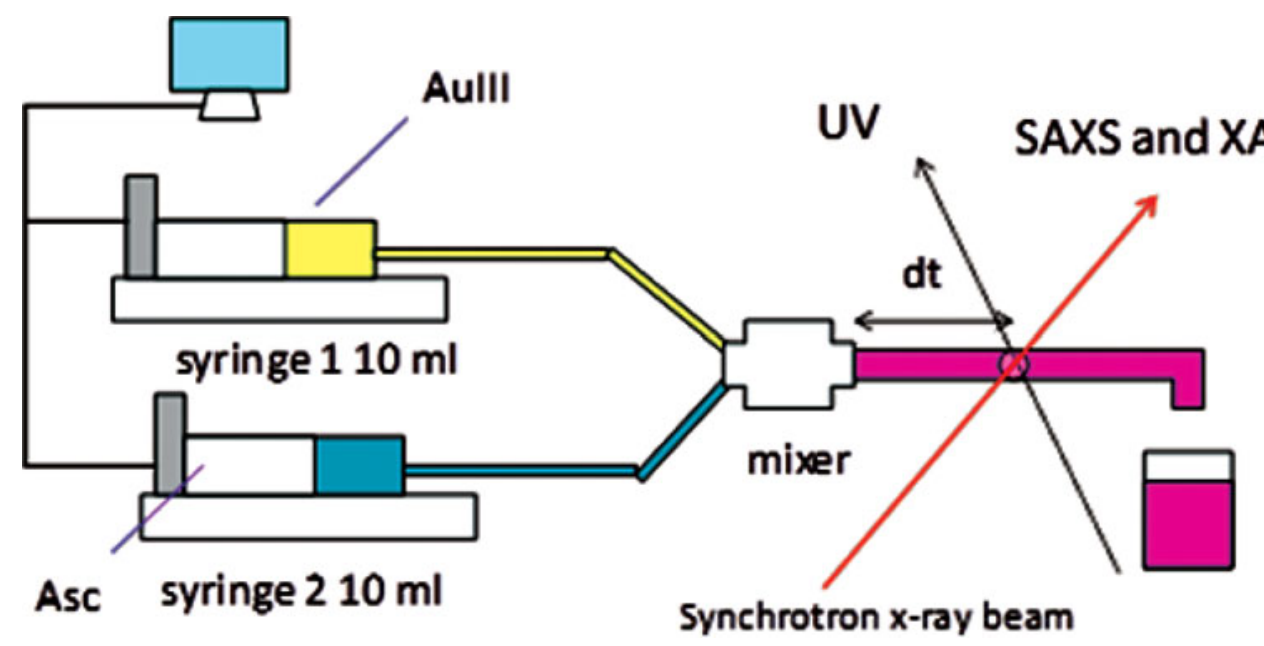

Figure 1. Systèmes de deux pousse-seringues alimentant un mélangeur millifluidique et capillaire permettant l'observation couplée SAXS/UV ou XANES/UV. Le temps d'observation après le mélange est contrôlé par la vitesse de poussée ; il varie typiquement dans la gamme 0.3-3 secondes.

en flux stoppé (appareil Biologic) pour les cinétiques très rapides (de l'ordre de la seconde) [2,3], soit en par un système de pousse-seringues couplé à un mélangeur et une ligne à retard avant le capillaire pour les cinétiques moyennement rapides, soit par un réacteur et une pompe péristaltique pour les réactions lentes (de l'ordre de la minutes [11] à quelques heures [4-6]). Ce texte présente plus en détail un travail concernant une synthèse effectuée en pousse-seringues (figure 1).

\subsection{Synthèse des nanoparticules}

\subsubsection{Synthèse de nanosphères par réduction par le borohydrure dans le toluène}

Les nanoparticules d'or sont obtenues par réduction $\mathrm{d}^{\prime} \mathrm{HAuCl}_{4}(3,5 \mathrm{mM})$ initialement solubilisé dans le toluène par du DDAB $(0,028 \mathrm{M})$. La réduction est effectuée par du borohydrure de sodium en large excès $(0,014 \mathrm{M})$ lui-même solubilisé dans une solution de toluène [2,3]. Un ligand stabilisant est également présent dans la solution réductrice à raison de $0,5 \mathrm{~mol} / \mathrm{L}$. Il s'agit soit d'acide décanoïque, soit de décylamine. Le mélange est assuré en 16 ms par un appareil de flux stoppé [SFM400-Biologic].

\subsubsection{Synthèse de nanosphères par réduction avec l'acide ascorbique}

Les nanoparticules d'or sont obtenues par synthèse en voie aqueuse sans ajout supplémentaire de tensioactifs pour assurer leur stabilité postérieure. La réduction du sel d'or $\left(\mathrm{HAuCl}_{4}\right)$ de concentration initiale allant de 0,5 $\mathrm{mM}$ à $6 \mathrm{mM}$ est assurée par de l'acide ascorbique [9] en léger excès ( $1 \mathrm{mM}$ à $12 \mathrm{mM})$. En effet l'équation bilan montre qu'il suffit de 1,5 molécules d'acide pour réduire l'Au(III) en $\mathrm{Au}(0)$. Le $\mathrm{pH}$ initial de la solution d'or s'étale de 3. à 2,2 suivant la concentration choisie. Le $\mathrm{pH}$ de la solution d'acide ascorbique peut être varié dans une large gamme. En effet, le contrôle de ce pH initial du réducteur permet de définir la taille finale des particules obtenues. C'est ce contrôle efficace que nous avons cherché à comprendre. La cinétique de réaction dépend du $\mathrm{pH}$ initial de l'acide ascorbique mais reste dans tous les cas extrêmement rapide ( 3 secondes pour les conditions les plus lentes). Un système de mélange rapide par pousse seringues tel que décrit dans la figure 1 a été utilisé dans ce cas. 


\subsubsection{Synthèse de nanobâtonnets par réduction avec l'acide ascorbique en présence de CTABr}

Lorsque l'on utilise l'acide ascorbique comme réducteur en présence d'un large excès de CTABr, ce tensioactif cationique créé un complexe avec l'or dans l'eau et l'acide ascrobique n'est plus capable de réduire l'Au(III) au-delà du stade Au(I). Ce complexe d'Au(I) reste parfaitement soluble dans l'eau. Par contre, l'ajout de BH4 déclenche alors la formation de quelques nucléi puis la croissance des particules. La dernière réduction est alors catalysée par la surface des particules en croissance.

Nous avons ainsi étudié la formation de bâtonnets d'or dans l'eau [10]. Ils sont obtenus par mélange de $4 \mathrm{mM}$ d'H+AuCl4- solubilisés dans l'eau en présence de 0,2 mol/L de CTABr et d'une quantité variable d'AgNO3 (0-2 mM) auquel sont successivement ajoutés de l'acide ascorbique (4,8 mM) puis une toute petite quantité d'BH4 $(5 \mu \mathrm{mol} / \mathrm{L})$. L'ajout d'acide ascorbique réduit l'Au(III) en une solution d'Au(I) parfaitement stable dans le temps. La croissance dure 20 minutes.

\subsection{Mesures des volumes, concentrations et tailles}

\subsubsection{Diffusion des rayons $X$}

Les diagrammes de diffusion aux petits angles ont été obtenus sur les lignes ID02 de l'ESRF (nanosphères dans le toluène et nanobâtonnets) et SWING de SOLEIL (nanosphères dans l'eau). Les temps de comptage était de $20 \mathrm{~ms}$ à $500 \mathrm{~ms}$ suivant la concentration. Un capillaire en quartz de 1,5 était utilisé comme cellule en ligne. Comme le système de nanoparticules est en croissance le nombre et le volume des nanoparticules varie au cours du temps. La fraction volumique de nanoparticules au cours du temps a ainsi été calculée en mesurant «l'invariant » au cours du temps en application des théorèmes généraux de la diffusion aux petits angles. Les concentrations et les distributions de taille au cours du temps ont été obtenues par des calages des spectres expérimentaux à partir de distribution de sphères gaussiennes.

\subsubsection{Absorption des rayons $X$ et $U V$}

L'ensemble des expériences d'absorption X près du seuil LIII de l'or ont été effectuées sur la ligne d'EXAFS dispersif ODE de SOLEIL. Due à la faible concentration d'or dans la solution (0.25-3 mM) réactive, une cellule présentant un chemin optique de $10 \mathrm{~mm}$ a été spécialement conçu afin d'obtenir des sauts d'absorbance de l'ordre de 0,1. De plus, les temps d'acquisition étant limitée à 13 ms par spectre (afin d'obtenir une résolution temporelle correcte lors d'une suivi continu), les expériences ont été répétées jusqu'à 100 fois et moyennées afin d'augmenter la qualité statistique de la mesure. Les mesures d'absorption UV ont plus classiquement été effectuées avec un spectrophotomètre fibré OCEAN Optic SD2000. Les temps d'acquisition étaient de $5 \mathrm{~ms}$. Dans ce dernier cas, le capillaire classique de 1,5 mm de diamètre était utilisé.

\section{RÉSULTATS ET DISCUSSION}

\subsection{Nanosphères dans le toluène}

Lors du suivi de la nucléation et croissance de ces objets, le mélange était assuré en 16 ms par un appareil de flux stoppé [SFM400-Biologic]. La réduction des ions Au(III) en ions solubles Au(I) se produit en environ $100 \mathrm{~ms}$ après le mélange, comme le montre les spectres d'absorption UV [2]. Par ailleurs, la diffusion centrale des rayons $\mathrm{X}$ apporte des renseignements complémentaires sur la morphologie des objets obtenus et sur leur cinétique de croissance. La figure 2 montre l'évolution au cours du temps de l'intensité diffusée par les nanoparticules en croissance.

La comparaison de la quantité d'Au(0) formé dans la solution obtenue par XANES avec la quantité d'or contenue dans les nanoparticules (SAXS), au cours du temps a permis dans ce cas de montrer que 

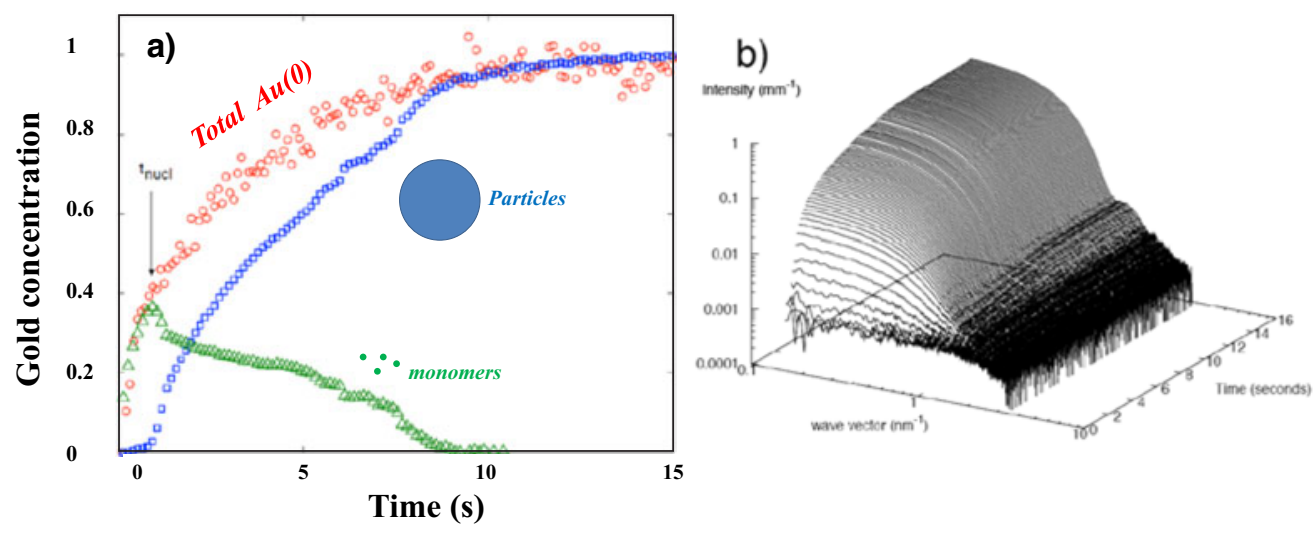

Figure 2. a) Comparaison du taux d'atomes d'or réduits au degré métallique (points rouges) et du taux d'atomes d'Au(0) compris dans les nanoparticules (points bleus); obtenus à partir de l'invariant des diagrammes de diffusion aux petits angles présentés en b). La différence (triangles verts) correspond aux atomes d'or encore en solution mais réduits à l'état métalliques $\mathrm{Au}(0)$. b) Formation des AuNP (en présence d'un ligand acide décanoïque) suivi in situ par SAXS. Diagrammes de diffusion en fonction du temps.

des atomes ou petits oligomères d'or existaient en solution en dehors des nanoparticules et ce pendant le temps transitoire de leur croissance. Un modèle numérique couplant la loi d'injection en monomère d'Au(0) avec les équations de nucléation et croissance permet de reproduire correctement les évolutions du nombre de particules et de leur taille au cours du temps [11]. Après une montée rapide initiale, le nombre de nanoparticules reste constant au cours de la croissance et la cinétique de réaction est dans ce cas limité par la réaction d'incorporation des atomes d'Au(0) aux nanoparticules.

\subsection{Nanospheres dans l'eau}

Le système millifluidique a été utilisé dans ce cas afin d'autoriser des temps de comptage de $500 \mathrm{~ms}$ en diffusion centrale des rayons $\mathrm{X}$ et de 2 secondes en absorption des rayons $\mathrm{X}$ tout en maintenant une résolution temporelle de $200 \mathrm{~ms}$. Ainsi la poussée est continue pendant le temps de comptage et le temps de réaction est défini par le retard entre le mélangeur et la cellule de détection. Les trois types d'expérience (UV/SAXS et XANES) ont été effectuées encore une fois en deux temps : les mesures UV et SAXS ont pu être couplées car utilisant le même capillaire. Les expériences de XANES devaient être faites sans UV, la cellule XANES ne permettant pas une observation UV en direction perpendiculaire au faisceau X. Parmi les résultats obtenus, la figure 4 montre la cinétique en UV. On observe clairement dans la région 200-300 nm la diminution des raies caractéristiques de l'Au(III) et de l'acide ascorbique. Cette diminution s'accompagne de l'apparition d'un pic plasmon à $520 \mathrm{~nm}$ qui constitue la signature des nanosphères d'or. L'analyse UV a permis de montrer que la consommation d' Au(III) était parallèle à la consommation d'acide ascorbique de manière monotone au cours du temps avec un rapport proche de la stoechiométrie attendue (1,5 AA pour un $\mathrm{Au}$ (III). La diffusion de rayonnement confirme la croissance à nombre constant de nanoparticules et ce d'autant plus rapidement que le pH est élevé. Ces conclusions sont en accord avec un mécanisme de nucléation croissance comparable à celui observé en toluène ; la diffusion et adhésion des monomères $(\mathrm{Au}(0))$ en suspension seraient les mécanismes dominants. La contribution des $\mathrm{Au}(\mathrm{I})$ comme intermédiaires potentiels (menant à un autre chemin réactionnel dans ce cas) reste cependant à évaluer. C'était le but d'une première expérience XANES en pousse seringues, non fructueuse car centrée sur les conditions diluées $(0,25 \mathrm{mM}$ d'Au(III)). Une seconde expérience à l'aide du nouveau détecteur rapide de la ligne ODE (résolution temporelle à la microseconde) et du 




Figure 3. Suivi UV-visible de la formation des nanosphères dans l'eau.

montage en flux stoppé a permis d'examiner le cas plus concentré $(3 \mathrm{mM})$ et est actuellement en cours de dépouillement.

\subsection{Nanobatônnets dans l'eau}

Les études XANES ont permis dans ce cas de démontrer une réduction de surface. Les spectres obtenus séquentiellement au cours des 20 minutes que dure la synthèse sont présentés en figure 4 . Le spectre initial correspond à de l'Au(I). Au cours du temps, chaque spectre peut être reproduit par un mélange d'Au(I) et d'Au( $(0)$. On peut alors déduire la fraction totale d'Au( 0$)$ dans l'échantillon. Elle est comparée dans l'insert à la fraction d' $\mathrm{Au}(0)$ contenue dans les nanoparticules (cette fraction étant obtenue à partir de l'invariant des spectres de diffusion aux petits angles (non montrés)). On constate que ces deux valeurs sont identiques. Ceci démontre qu'il n'y a pas d' $\mathrm{Au}(0)$ en dehors des nanoparticules en contraste avec le cas précédent du mécanisme de formation des nanosphères dans le toluène. Les expériences de XANES permettent donc de démontrer définitivement que la dernière étape de réduction d'Au(I) en $\mathrm{Au}(0)$ a lieu uniquement à la surface des particules en croissance [11] confirmant l'hypothèse de catalyse de surface supposée depuis 5 ans pour ce système. La diffusion centrale des rayons a par ailleurs permis de montrer que la croissance anisotrope en nanobâtonnets était due à une vitesse de dépôt plus rapide sur les pointes que sur les faces latérales des objets. Le ralentissement latéral pourrait être du à une meilleure compacité de la bicouche de tensioactifs absorbée sur les faces latérales que sur les points, mais cette hypothèse reste à confirmer. 


\section{Web of Conferences}

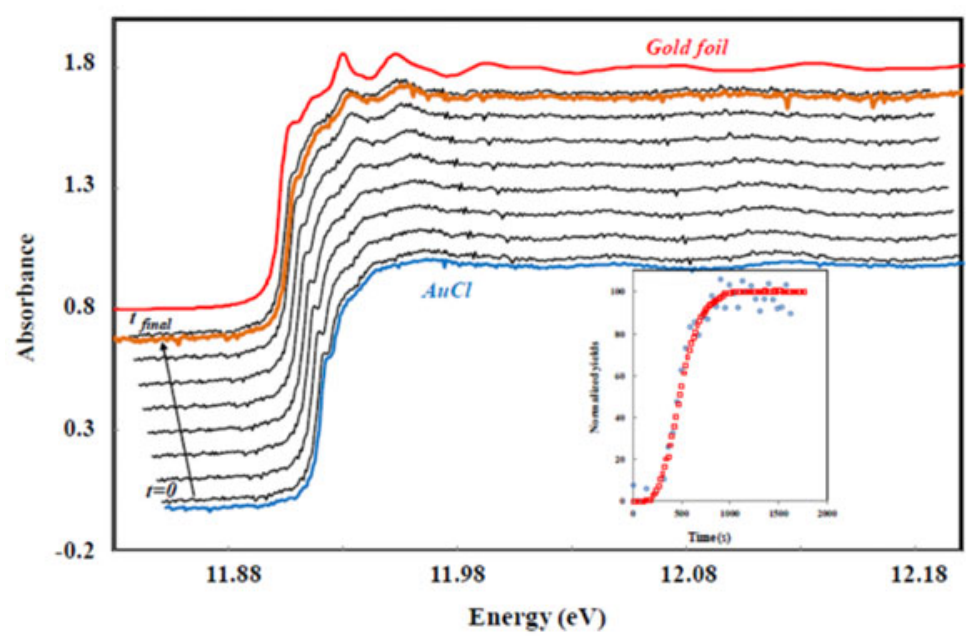

Figure 4. Évolution au cours du temps près du seuil LIII de l'or lors de la formation de nanobâtonnets.

\section{CONCLUSIONS}

L'utilisation de méthodes de caractérisation résolues en temps pour la compréhension des mécanismes de croissance de nanoparticules est devenue accessible grâce à l'avènement des synchrotrons de troisième génération. Un spectre d'adsorption $\mathrm{X}$ peut aujourd'hui techniquement être acquis en quelques millisecondes sur les lignes dispersives. De même, un diagramme de diffusion aux petits angles requiert 10-5 ms d'acquisition et permet de suivre des croissances à l'échelle de la seconde. Ainsi, il apparait que les outils expérimentaux sont parfaitement prêts. La difficulté réside finalement plus dans le choix de la chimie de synthèse pouvant être examinée dans un cas suffisamment modèle pour être interprétable et dans la capacité des chimistes et physiciens à s'associer pour développer les analyses adhoc de ces systèmes réactifs à multiples changements d'échelle.

\section{Références}

[1] Y. Xia, Y. Xiong, B. Lim, S.E. Skrabalak, Angew. Chem. Int., 48, 60-103 (2009)

[2] B. Abécassis, F. Testard, O. Spalla, P. Barboux, Nanoletters, 7-(6) 1723-1727 (2007)

[3] B. Abécassis, F. Testard, O. Spalla, Phys. Rev. Lett., 10011115504 (2008)

[4] S. Fouilloux, A. Désert, O. Taché, O. Spalla, J. Daillant, A. Thill, J. Colloid Interface Sci., 436(1) 79-86 (2010)

[5] S. Fouilloux, O. Taché, O. Spalla, A. Thill, Langmuir, 27 (20) 12304-12311 (2011)

[6] P. Maillet, C. Levard, O. Spalla, A. Masion, J. Rose, A. Thill, Phys. Chem. Chem. Phys., 13 2682-2689 (2011)

[7] M. Harada, H. Einaga, Langmuir, 236536 (2007)

[8] J. Polte, T.T. Ahner, F. Delissen, S. Sokolov, F. Emmerling, A.F. Thünemann, R. Kraehnert, J. Am. Chem. Soc., 132 (4) 1296-1301 (2010)

[9] D. Andreescu, T.K. Sau, D.V. Goia, J. Colloid Interface Sci., $298742-751$ (2006)

[10] F. Hubert, F. Testard, A. Thill, Q. Kong, O. Tache, O. Spalla, Crystal Growth \& Design, 12 1548-1555 (2012)

[11] B. Abécassis, F. Testard, Q. Kong, F. Baudelet, O. Spalla, Langmuir, 2613847 (2010) 